\title{
ON HECKE'S FUNCTIONAL EQUATION
}

\section{BY K. CHANDRASEKHARAN AND RAGHAVAN NARASIMHAN}

Communicated by S. Bochner, August 26, 1960

Let $\left\{\lambda_{n}\right\},\left\{\mu_{n}\right\}$ be two strictly increasing sequences of positive numbers, and $\left\{a_{n}\right\},\left\{b_{n}\right\}$ be two sequences of complex numbers not identically zero, and $s$ a complex variable with $s=\sigma+i t$. Let $\delta$ be a real number. The functions $\phi, \psi$, representable as $\phi(s)=\sum a_{n} \lambda_{n}^{-s}$, $\psi(s)=\sum b_{n} \mu_{n}^{-s}$ are said to satisfy the functional equation

$$
(2 \pi)^{-s} \Gamma(s) \phi(s)=(2 \pi)^{s-\delta} \Gamma(\delta-s) \psi(\delta-s),
$$

if there exists in the $s$-plane a domain $D$ which is the exterior of a bounded, closed set $S$, and in $D$ there exists a holomorphic function $\chi(s)$ with the property

$$
e^{-\epsilon t} \chi(\sigma+i t)=O(1), \quad 0<\epsilon<\pi / 2,
$$

as $|t| \rightarrow \infty$, uniformly in each strip $\sigma_{1} \leqq \sigma \leqq \sigma_{2},-\infty<\sigma_{1}<\sigma_{2}<+\infty$, and

$$
\begin{array}{ll}
\chi(s)=(2 \pi)^{-s} \Gamma(s) \phi(s), & \text { for } \sigma>\alpha, \\
\chi(s)=(2 \pi)^{s-\delta} \Gamma(\delta-s) \psi(\delta-s), & \text { for } \sigma<\beta,
\end{array}
$$

where $\alpha, \beta$ are some constants [1].

THEOREM 1. (A) Functional equation (1) implies the identity

$$
\begin{aligned}
& \frac{1}{\Gamma(\rho+1)} \sum_{\lambda_{n} \leq x}^{\prime} a_{n}\left(x-\lambda_{n}\right)^{\rho} \\
& \quad=\left(\frac{1}{2 \pi}\right)^{\rho} \sum_{n=1}^{\infty}\left(\frac{x}{\mu_{n}}\right)^{(\delta+\rho) / 2} b_{n} J_{\delta+\rho}\left\{4 \pi\left(\mu_{n} x\right)^{1 / 2}\right\}+Q_{\rho}(x),
\end{aligned}
$$

for $x>0$, and $\rho \geqq 2 \beta-\delta-1 / 2$, where $\beta$ is a number for which $\sum_{1}^{\infty}\left|b_{n}\right| \mu_{n}^{-\beta}<\infty$, and $Q_{p}$ is a "residual function" given by

$$
Q_{\rho}(x)=\frac{1}{2 \pi i} \int_{\mathcal{C}} \frac{\chi(s)(2 \pi)^{s} x^{s+\rho}}{\Gamma(\rho+1+s)} d s,
$$

where $\mathfrak{e}$ denotes any curve, or curves, encircling all of $S$.

Conversely, identity (2) implies functional equation (1).

(B) Functional equation (1) implies the identity

$$
\begin{aligned}
& \left(-\frac{1}{s} \frac{d}{d s}\right)^{\rho}\left[\frac{1}{s} \sum_{n=1}^{\infty} a_{n} e^{-s} \lambda_{n}^{1 / 2}\right] \\
& \quad=2^{s \delta+\rho} \Gamma\left(\delta+\rho+\frac{1}{2}\right) \pi^{\delta-1 / 2} \sum_{n=1}^{\infty} \frac{b_{n}}{\left(s^{2}+16 \pi^{2} \mu_{n}\right)^{\delta+\rho+1 / 2}}+R_{\rho}(s),
\end{aligned}
$$


where

$$
R_{\rho}(s)=\frac{1}{2 \pi i} \int_{\mathcal{C}} \frac{\chi(z)(2 \pi)^{z} \Gamma(2 z+2 \rho+1) 2^{-\rho}}{\Gamma(z+\rho+1)} s^{-2 z-2 \rho-1} d z
$$

for $\operatorname{Re} s>0, \rho$ integral, $\rho>-\delta-1 / 2$, and $\rho \geqq 2 \beta-\delta-1 / 2$. If $\beta>0$, then (4) holds for $\rho$ integral and $\rho \geqq \beta-\delta-1 / 2, \rho \geqq 0$.

Conversely, if identity (4) holds for some $\rho$ which is integral, and such that $\sum_{n=1}^{\infty}\left|b_{n}\right| \cdot \mu_{n}^{-\delta-\rho-1 / 2}<\infty$, then we have functional equation (1).

A sharper form of identity (2) is proved by an appeal to a theorem of Zygmund [7] on the equi-convergence of trigonometric integrals.

Theorem 2. Suppose that

$$
\sum_{n=1}^{\infty} \frac{\left|b_{n}\right|}{\mu_{n}^{\beta}}<\infty,
$$

and

$$
\sup _{0 \leqq h \leqq 1}\left|\sum_{m^{2}<\mu_{n} \leq(m+h)^{2}} \frac{b_{n}}{\mu_{n}^{\beta-1 / 2}}\right|=o(1) \text {, as } m \rightarrow \infty \text {, }
$$

and that

$$
\text { functional equation (1) holds. }
$$

Then the series of Bessel functions on the right hand side of (2) converges for $\rho \geqq 2 \beta-\delta-3 / 2$, uniformly in any interval in $x>0$, in which the function on the left hand side of (2) is continuous, and boundedly in any interval $0<x_{1} \leqq x \leqq x_{2}<\infty$ if $\rho=0$.

Using Zygmund's extension [7] of his equi-convergence theorem into an equi-summability theorem, the following result is proved.

Theorem 3. Suppose in addition to conditions (5), (6) and (7) that $\mu_{n}<c \cdot n$, for some constant $c>0$. Then the series

$$
\sum_{n=1}^{\infty} b_{n}\left(\frac{x}{\mu_{n}}\right)^{(\delta+\rho) / 2} J_{\delta+\rho}\left\{4 \pi\left(\mu_{n} x\right)^{1 / 2}\right\}, \quad x>0,
$$

is summable by Cesaro's means of order $k=\max (2 \beta-\delta-3 / 2-\rho, 0)$. The summability is uniform in every finite interval of continuity of $(1 / \Gamma(\rho+1)) \sum_{\lambda_{n}<x} a_{n}\left(x-\lambda_{n}\right)^{\rho}$ in $x>0$. And the sum is $(1 / \Gamma(\rho+1))$ - $\sum_{\lambda_{n}<x} a_{n}\left(x-\lambda_{n}\right)^{\rho}-Q_{\rho}(x)$ if $\rho$ is not a negative integer, and $-Q_{\rho}(x)$ if $\rho$ is a negative integer, where $Q_{-n}(x)$ is the $(m+n)$ th derivative of $Q_{m}(x)$ for $m$ large.

Theorems 1, 2 and 3 are used to obtain, in their sharpest form, 
several identities involving arithmetical functions such as Ramanujan's $\tau(n)$, or the function $r_{k}(n)$ giving the number of lattice points on a $k$-dimensional sphere, or the function $\sigma_{k}(n)$ giving the sum of the $k$ th powers of the divisors of $n$, where $k$ is an odd integer, or $\mu(S, t)$ which is Siegel's measure of representation of a number $t$ by $S$, where $S$ is an indefinite quadratic form in more than 4 variables, with rational coefficients and positive determinant, since such functions occur as the coefficients of Dirichlet series which satisfy functional equation (1). In particular, the identity of the form (2) obtained for $\tau(n)$ is sharper than that of Wilton and Hardy $[6 ; 2]$.

By generalizing identity (4) in an appropriate manner, and applying the methods of A. E. Ingham $[3 ; 4]$, we prove the following results on the error term in the average order of a class of arithmetical functions.

THEOREM 4. Suppose that functional equation (1) holds, and that the sequence $\left\{\lambda_{n}\right\}$ contains a subset $\left\{\lambda_{n_{k}}\right\}$ such that no number $\lambda_{n}^{1 / 2}$ is representable as a linear combination of the numbers $\lambda_{n_{k}}^{1 / 2}$ with coefficients \pm 1 , unless $\lambda_{n}^{1 / 2}= \pm \lambda_{n_{r}}^{1 / 2}$ for some $r$, in which case $\lambda_{n}^{1 / 2}$ has no other representation. Suppose, in addition, that

$$
\sum_{k=1}^{\infty} \frac{\left|\operatorname{Re} a_{n_{k}}\right|}{\lambda_{n_{k}}{ }^{(\delta+\rho) / 2+1 / 4}}=+\infty, \quad \rho \geqq 0 .
$$

Then

$$
\limsup _{x \rightarrow \infty} \frac{\operatorname{Re}\left[B_{\mu}^{\rho}(x)-Q_{\rho}(x)\right]}{x^{\delta / 2+\rho / 2-1 / 4}}=+\infty
$$

and

$$
\liminf _{x \rightarrow \infty} \frac{\operatorname{Re}\left[B_{\mu}^{\rho}(x)-Q_{\sigma}(x)\right]}{x^{\delta / 2+\rho / 2-1 / 4}}=-\infty
$$

where

$$
B_{\mu}^{\rho}(x)=\sum_{u_{n} \leq x}^{\prime} b_{n}\left(x-\mu_{n}\right)^{p} .
$$

Theorem 5. If functional equation (1) holds, and $\operatorname{Re} a_{n} \neq 0$ for at least one value of $n$, then

$$
\limsup _{x \rightarrow \infty} \frac{\operatorname{Re}\left[B_{\mu}^{\rho}(x)-Q_{\rho}(x)\right]}{x^{\delta / 2+\rho / 2-1 / 4}}>0
$$


and

$$
\lim _{x \rightarrow \infty} \frac{\operatorname{Re}\left[B_{\mu}^{\rho}(x)-Q_{\rho}(x)\right]}{x^{\delta / 2+\rho / 2-1 / 4}}<0,
$$

whereas if $\operatorname{Im} a_{n} \neq 0$ for at least one value of $n$, we have

$$
\limsup _{x \rightarrow \infty} \frac{\operatorname{Im}\left[B_{\mu}^{\rho}(x)-Q_{\rho}(x)\right]}{x^{\delta / 2+\rho / 2-1 / 4}}>0
$$

and

$$
\lim _{x \rightarrow \infty} \inf \frac{\operatorname{Im}\left[B_{\mu}^{\rho}(x)-Q_{\rho}(x)\right]}{x^{\delta / 2+\rho / 2-1 / 4}}<0 .
$$

The exponent of $x$ in (8) and (9) is the "best possible" for sufficiently large $\rho$, in the sense that for such $\rho$ we have

$$
B_{\mu}^{\rho}(x)-Q_{\rho}(x)=O\left(x^{\delta / 2+\rho / 2-1 / 4}\right) .
$$

Theorems 4 and 5 apply, in particular, to the arithmetical functions mentioned above. Theorem 4 , for instance, gives for $\rho=0$ the results of Ingham [3] and Pennington [5] for $r_{2}(n)$ and $\tau(n)$. Full details and proofs will be published elsewhere.

\section{BIBLIOGRAPHY}

1. S. Bochner, Some properties of modular relations, Ann. of Math. vol. 53 (1951) pp. 332-363.

2. G. H. Hardy, A further note on Ramanujan's arithmetical function $\tau(n)$, Proc. Cambridge Philos. Soc. vol. 34 (1938) pp. 309-315.

3. A. E. Ingham, On two classical lattice point problems, Proc. Cambridge Philos. Soc. vol. 36 (1940) pp. 131-138.

4. - On two conjectures in the theory of numbers, Amer. J. Math. vol. 64 (1942) pp. 313-319.

5. W. B. Pennington, Order of magnitude of Ramanujan's arithmetical function $\tau(n)$, Proc. Cambridge Philos. Soc. vol. 47 (1951) pp. 668-678.

6. J. R. Wilton, A note on Ramanujan's arithmetical function $\tau(n)$, Proc. Cambridge Philos. Soc. vol. 25 (1929) pp. 121-129.

7. A. Zygmund, On trigonometric integrals, Ann. of Math. vol. 48 (1947) pp. 393-440.

Tata Institute of Fundamental Research,

BOMBAY, INDIA 